\title{
Hand-around on Seamless Services and Mobility in Home Networking
}

\author{
Pei-Yuan Qiu', Min-Shu Hung ${ }^{1}$, Jen-yi Pan', \\ Kuo-Pao Fan', and Jui-Wen Chen ${ }^{2}$ \\ 'Department of Communications Engineering National Chung Cheng University of Taiwan \\ dimhdp10@ant.comm.ccu.edu.tw \\ hungenter@hotmail.com \\ jypan@comm.ccu.edu.tw \\ ${ }^{2}$ Industrial Technology Research Institute of Taiwan \\ \{kpfan, RyanChen\}@itri.org.tw
}

\begin{abstract}
In the age of technology, most of the network services originally run on large scale and base on complicated systems. When they're brought into our home, the migration could derive further technical problems due to the change of scale and cost. Home networking structure is often improvised and built up with several types of wireless technology, such as WiMedia and WiFi, inside several small rooms. In addition, people enjoy walking around at home and being served without constraints and service interruption, so the seamless services and mobility within home are a must. This paper describes a novel notion on the transition among wireless connectivities at home which is named Hand-around and efficiently provides seamless network services while moving in heterogeneous and improvised home networking. A developing technique in Mobile IPv6, called multiple care-of addresses $(\mathrm{MCoA})$ registration, is the best candidate to accomplish such seamless services in an all-IP home network. Besides, it's a pure layer3 mechanism and hence could more easily apply on and adapt to home services without massively changing the operational flow and system hierarchy, especially suited for services of multimedia, communication and entertainment. This notion also gives a new vision to some scenarios described in Home Gateway Initiative (HGI) document.
\end{abstract}

Keywords: Home networking, Home gateway, Seamless service, Mobile home service, Multiple care-of address registration, Heterogeneous networks. 


\section{Introduction and Background}

Nowadays, the performance of personal computers has been improved day by day, and the broadband network has also been introduced into home environments. These advancements have created a new type of service, which is inside a house serving just few people, rather than most internet applications that connect to a respectable number of users. It is important to bring the convenience and comfort of these applications right into home, after a day of working, and can even create new services like media center, and home IP-PBX, which provides an IP-based telephone system. That's what we called digital life is. This kind of service usually relies on many devices working simultaneously, including embedded systems and personal computers. These devices can be divided into three categories: the devices providing broadband, the devices providing the connectivity between devices, and the devices working for services in home networking. Figure 1 illustrates the location of digital devices inside a house. These devices are serving in their corresponding places. For example, there are televisions in the living room and parents' room. According to their requirements and characteristics, the devices have various kinds of connectiveties, like wireless headphones using Bluetooth [1], and laptops using $\mathrm{Wi}-\mathrm{Fi}[2]$. Hence, inside the house there may have diverse wireless connectivities, such as Wi-Fi, Bluetooth, ZigBee [3], IrDA [4], wireless USB [5], WiMedia [6], and so on. They may be deployed casually and without comprehensively planned so their coverage could be heavily overlapping. We've got convenience by using these wireless services, but uncertainties exist when it comes to mobility, especially on handoff issues.

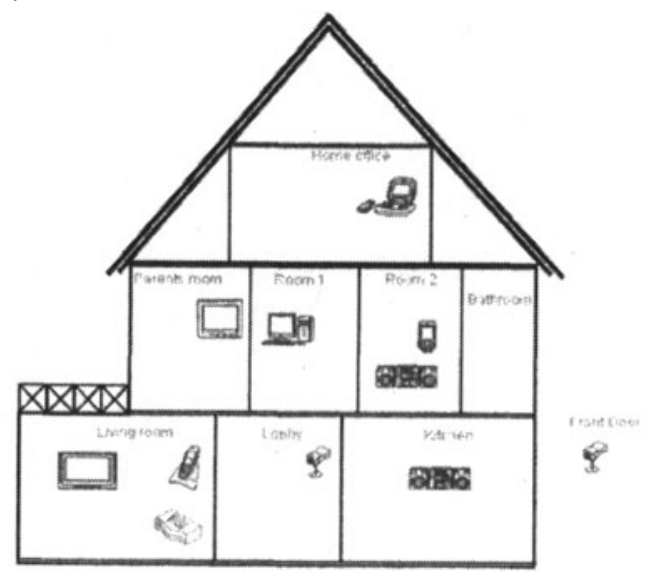

Fig. 1. Home [7] 


\section{Home Networking Services with Mobility}

Following subsections list some services that might have mobility concerns in HGI [7].

\subsection{Communication}

Voice over IP (VoIP) [8] is the main communication application. Many VoIP enduser device products have Wi-Fi or other wireless connectivity, and a laptop could be a VoIP client device, too. By carrying one of these devices, users could walk through doors from room to room while talking on VoIP. So, mobility is now a necessary part of communication at home.

\subsection{Fix Mobile Convergence}

Fix mobile convergence is based on the premise that the device has multiple network interfaces. For example, an unlicensed mobile access (UMA) [9] phone may have both GSM and Wi-Fi radio module. Also there are $3 \mathrm{G} / \mathrm{Wi}-\mathrm{Fi}$ scenarios that can switch to VoIP in home to reduce some expense. To seamlessly switch from one network to another without cutting off a living session needs a hand-off mechanism carried with mobility.

\subsection{Entertainment \& Information}

\subsubsection{Multimedia}

Multimedia enjoyments are common entertainment in modern lives, especially at home, people expect to get some relax by listening to music, watching videos, or many other multimedia clips. Watching multimedia clips without lags when users roam around at home can be a relaxation after a whole day's working, and it can be achieved by carrying a playing device with multimedia contents stored inside. But when the contents or multimedia streams are on the Internet or simply in a home media center which cannot be easily carried about, the media continuity is certainly a different story especially when the users move around from a wireless coverage to another. 


\subsubsection{Gaming}

Gaming is an industry growing explosively; it's about gaming type, platform, contents, multi-sense presentation, and also how players make control commands. Cordless game controllers have become burning to the touch instead of wired controllers. If the control commands can be transmitted via network to the game console with mobility and seamless design, the "gaming everywhere at anytime" dream, i.e. pervasive gaming [10], can be accomplished.

\subsubsection{IPTV}

IPTV [11] is a real-time delivery that broadcasts quality TV over IP. IPTV is also a kind of multimedia application. But with stricter quality definition and other extensions, such as encryption, multi-stream capability and IP-core design, bringing IPTV into mobility and keeping the contents played seamlessly creates technically issues.

\subsection{Home Management \& Security}

Some home management and security devices also have wireless connectivity. A universal remote control must have the ability to access home network at every position at home. Also, there may have monitoring cameras moving inside the house. For example, a camera attached to a vivacious baby could help adults keep a watch on her. And of course the video content must be seamless. 


\section{Discussion of Handoff and Mobility Scenario}

Here we discuss a scenario about mobile IP in home networking.

Cell phones are now small but powerful. There's a trend that they're equipped with several network interfaces including $3 \mathrm{G} / \mathrm{GSM}$, Wi-Fi, Bluetooth, IrDA, even WiMAX in a near future. Although the original design of mobile communicating function uses only $3 \mathrm{G} / \mathrm{GSM}$, but with VoIP technology, using other interfaces is possible and already commercialized, such as UMA.
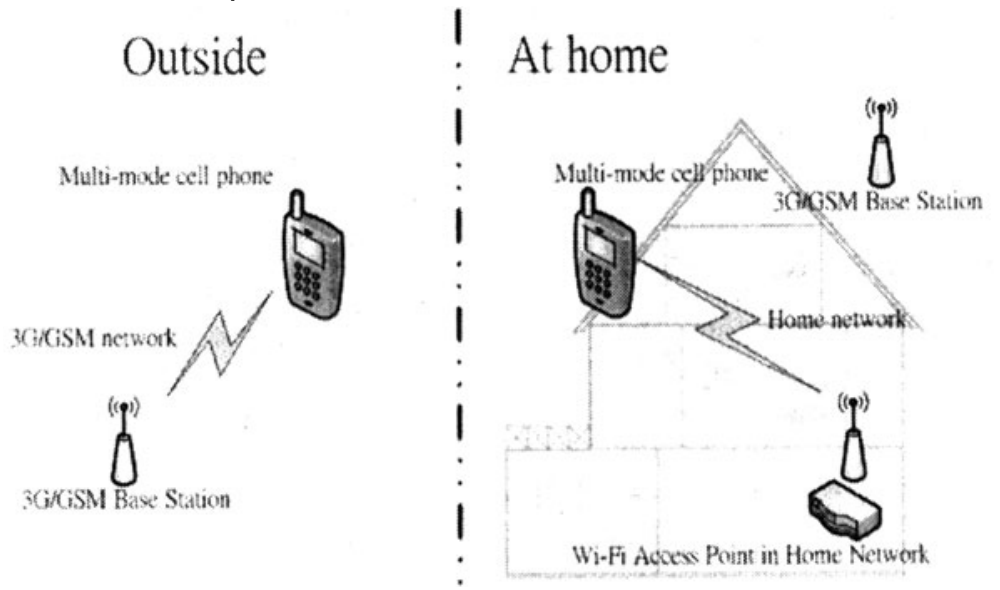

Fig. 2. Multi-mode Cell Phone

Fig. 2 shows the basic principle of the multi-mode cell phone. When in outdoors or somehow without other connectivity except 3G/GSM, a multi-mode cell phone works just like a single-mode one. However, when it's at home, it can establish several kinds of connections to the home network, and uses them to replace 3G/GSM on communicating purpose. The benefit is, when using non-3G/GSM network, the message flow goes through an IP-core network, communicating using VoIP. VoIP is considered cheaper than 3G/GSM, since the physical linkage is the home broadband instead of mobile networks. Also, when at home, that is, inside a modern building, the 3G/GSM signal quality may not be capable of communication, so switching to another network with stronger connectivity would be better. Nevertheless, signal quality is still an issue regardless of $3 \mathrm{G} / \mathrm{GSM}$. Because the wireless base stations (BS) or access points (AP) inside our home are usually deployed without projections, the multi-mode cell phone must have the ability to choose the right interface with better signal quality. Hence, handoff is still necessary even inside our home. 


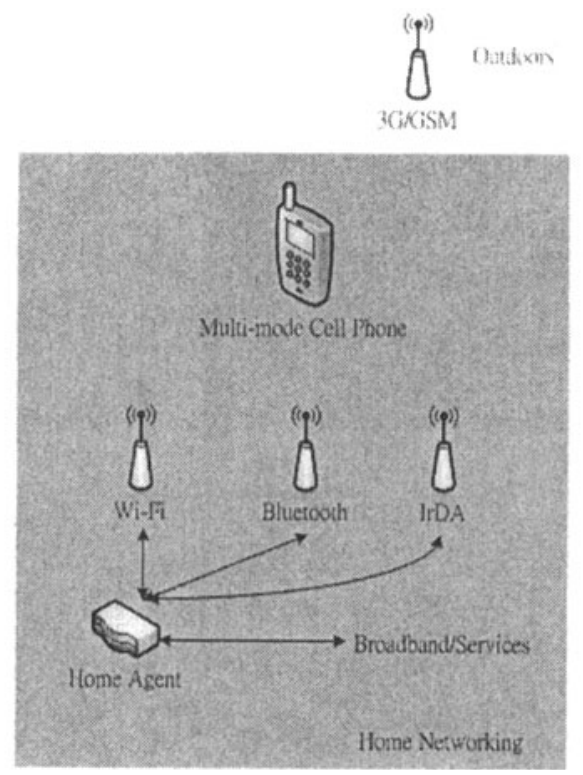

Fig. 3. Accessible Communicating Base Stations at Home

Fig. 3 illustrates the possible physical connections at home. Since signal quality may be altering when the multi-mode cell phone is moving, selection between these base stations would be necessary. Hence, a call session may maintained by switching from these connectivity. The handoff technology in 3G/GSM network has already come to maturity, but there's no widely acceptable handoff technology between these indoor connectivity based on IP-core network. Besides, the demand for communication quality is rigid when it comes to commercial services. Can VoIP handoff achieve seamless? 


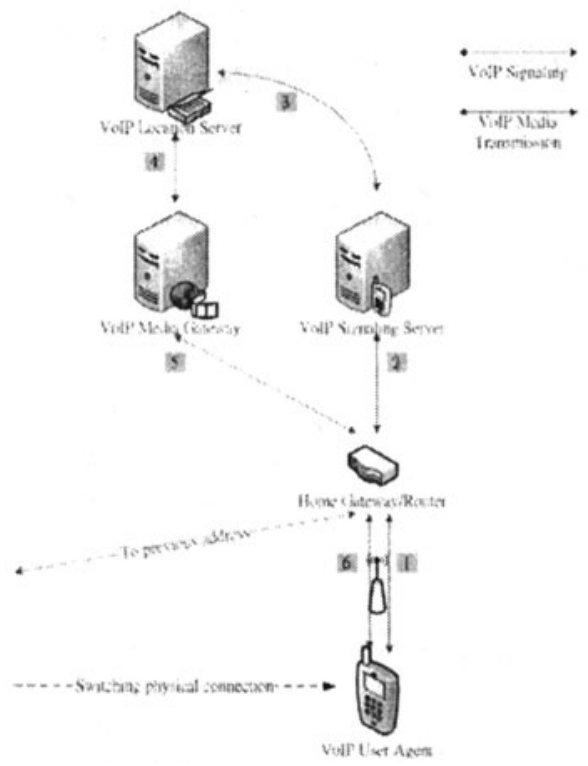

Fig. 4. VoIP Call Forwarding

A VoIP system must have call forwarding ability, and call forwarding can be a possible way to deal with handoffs between IP-core connections [12]. As illustrated in Fig. 4, a VoIP call forwarding action takes six procedures:

- Step 1 and step 2 : When switching connection, VoIP user agent informs the VoIP signaling server via the home gateway/router that the address of the VoIP user agent had been changed.

- Step 3 : Updating location information.

- Step 4 : Looking up new location.

- Step 5 and step 6 : The VoIP media gateway redirects the communication content again to reach the new address of the VoIP user agent.

However it's not a good solution at all. Since VoIP is based on application layer protocols, and the user agent informs the VolP system after the active physical connection is changed, call forwarding would take a lot of time, and causes interruption in call sessions. That's not acceptable with commercialized telephone services. 


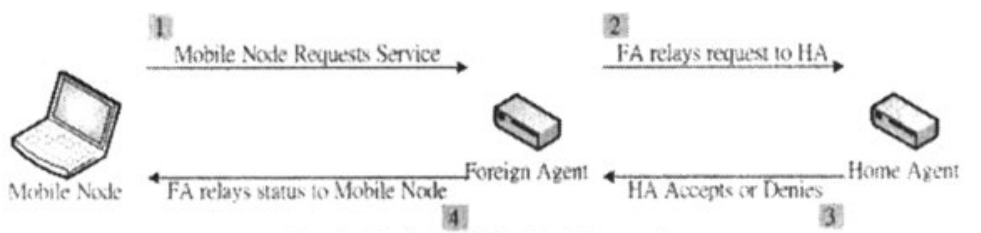

Fig. 5. Basic Mobile IPv4 Scenario

Fig. 5 is a basic scenario of mobile IPv4 (MIPv4). When a mobile node (MN) moves to a foreign network, it first send a request to the foreign agent, and then the foreign agent relays the request to the home agent of the mobile node. The home agent (HA) tells foreign agent (FA) whether the request is accepted or denied, and then the foreign agent relays the decision to the mobile node.

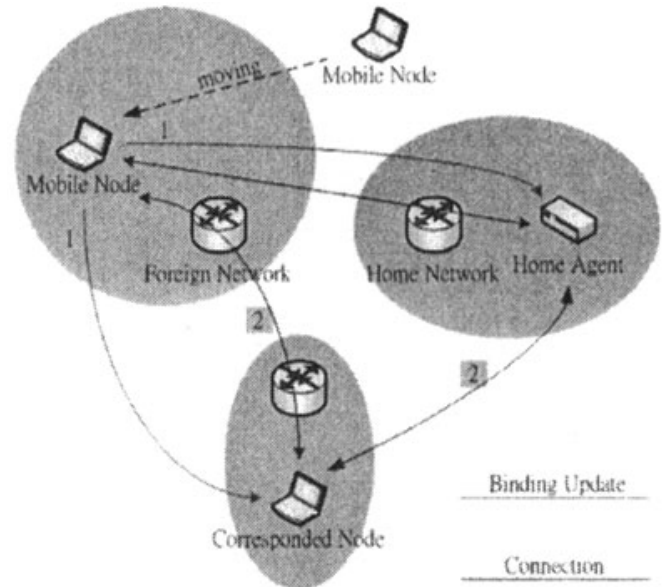

Fig. 6. Basic Mobile IPv6 Scenario

As shown in Fig. 6, the biggest difference between mobile IPv4 and mobile IPv6 (MIPv6) is that the MIPv6 does not require foreign agent because the router of the foreign network can assist the MN to form the care-of address, which is done by foreign agents in MIPv4. When a MN travels to a foreign network, it first gets a care-of address $(\mathrm{CoA})$, and then the $\mathrm{MN}$ send binding update $(\mathrm{BU})$ directly to the home agent and correspondent node $(\mathrm{CN})$. So both HA and $\mathrm{CN}$ know the $\mathrm{CoA}$ of the MN. Thus, $\mathrm{CN}$ and MN may connect to each other with or without the tunneling by HA.

Mobile IP could be a solution. Since the connections are all based on IP-core network, the multi-mode cell phone must have an IP address. When handoff occurs, the VoIP user agent may still need to follow these procedures, but the home agent knows it. The home agent knows it right away when the VoIP user agent is taking a physical handoff changing the IP address, since mobile IP technology is a layer-3 method. So the home agent just simply tunnels the communicating media content to the new address regardless of the VoIP call forwarding. There's no 
longer need to wait for the VoIP system passively accepting requests from VoIP user agents and then taking VolP switching procedures. Even if the VoIP system does no forwarding procedures, as long as the home agent handles the tunneling, the call session can be maintained. This method could avoid the interruption during call forwarding by VoIP system and satisfy users' expectation on voice quality.

We can learn from this VolP scenario that by switching to another connection, applications could be effected and need to be recovered. Mobile IP is a layer- 3 solution, which could be better than other solutions of upper-layers in the aspect of application independence, i.e. suiting any application without modification. A mobile IP environment is usually composed with good schemes and constructions. With wide coverage and low overlapping, the capitalized cost, the frequency of handoff, the signaling effect, all could be reduced. However, in home networking environment, wireless coverage is usually highly overlapped and with uncertainty due to the house-building structures. So handoffs could occur frequently, and uncertainties exist on when and how to do vertical handoffs, and how to determine the best foreign network since several kinds of foreign networks may be detected. These are the differences between traditional mobile IP and home mobile IP environment. Also, the handoff latency should be considered. As shown in Fig. 5 and Fig. 6, a handoff action consists of several steps, and the latency cannot be ignored. Now we address a notion named hand-around to cope with these concerns. 


\section{Hand-around}

In this section, we will start from the motivation and definition of handaround. Then we will discuss some issues and possible challenges in handaround. At last, we will compare several techniques of connectivity transition and illustrate that Multiple Care-of Addresses registration (MCoA), which was produced by the IETF working group "Mobile Nodes and Multiple Interfaces in IPv6" (Monami6) [13], should be the most proper layer 3 mechanism for connectivity transition in heterogeneous wireless home networks with highly overlapped coverage per our simulation.

\subsection{Motivation and definition}

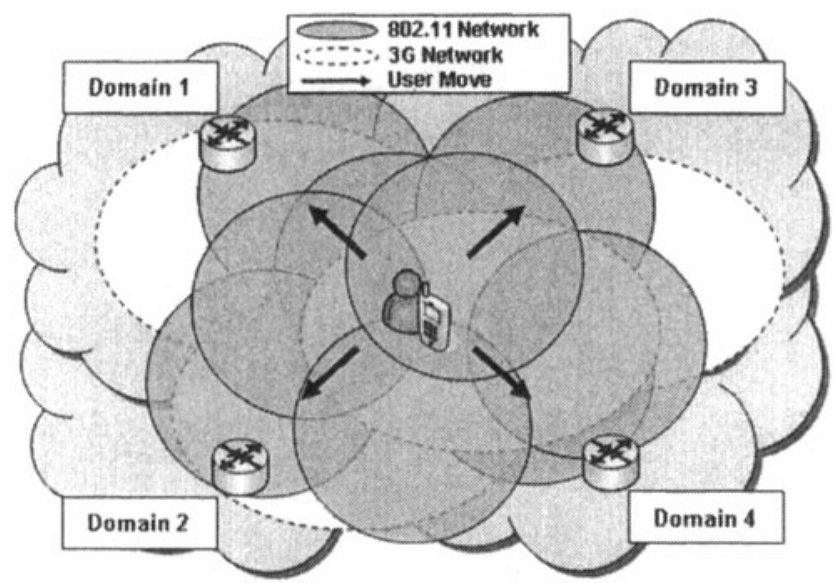

Fig. 7. Heterogeneous wireless networks with highly overlapped coverage [14]

Considering the scenario in Fig. 7, MN has multiple interfaces. The MN locates in wireless networks with heterogeneous layer 2 technologies and several IP domains. We observe that in this dense overlapping network the $\mathrm{MN}$ always has at least one interface staying connected while other interfaces' associations transit from one access point (or base station) to another, and thus keeps continuous online with hardly any disconnection caused by weak signals or connectivity transition. In this situation handoff latency nearly disappears according to the usual definition (i.e., the period between the last moment before disconnection and the next successful data transmission). We name this process of connectivity transition where at least one interface keeps working and hence the $\mathrm{MN}$ keeps continuous online without interruption caused by other interfaces' transition, as handaround. Here are some observations and features about hand-around:

1. More than one IP domains probably overlap. 
2. Base stations locate in high density, and wireless coverage areas are highly overlapped.

3. Users can transmit data through one or more interfaces concurrently.

In fact, many research projects and operators continuously develop related seamless integration technology of these networks with high degree of heterogeneity and highly overlapped coverage. For example in Ambient network [15, 16], users can have the best choice when the network condition varies in difference places, time, and even network loading. As fore-mentioned, the UMA is also a good example of such seamless integration technology. Hand-around already affects our daily life, and traditional assumption about handoffs should be reviewed. Some points of view in such environment will be discussed in the next subsection.

\subsection{Discussion}

We discuss several points about hand-around as follows.

- Construction and Decision: Formerly wireless network construction proceeds from using minimum number of base stations for maximum coverage, but it should be changed in heterogeneous wireless networks with highly overlap coverage, e.g., houses and hotels. Heavy-load sharing, seamless services or better transmission quality could be the primary consideration. Just maintaining access to Internet is not the only purpose in user's point of view. Issues like how to choose a suitable wireless network by user preference or enhance transmission efficiency by multi-interface are more important during handaround.

- Handoff Target and Timing: We propose a new viewpoint of connectivity transition, called hand-around, which is different from traditional handoff. Handaround doesn't specify a target access point or base station to attach to but a set of simultaneous access points or base stations during connectivity transition because of concurrent activations of multiple interfaces and dense overlap of heterogeneous wireless serving areas. Hand-around also doesn't identify specific timing and location of connectivity transition, but traditional handoffs do. In hand-around, the $\mathrm{MN}$ with the support of multiple interfaces, which activate at the same time and have at least one connected to base station or access point, can keep continuous transmission regardless of some interfaces proceeding transition from one access point or base station to another, and therefore the handoff latency doesn't need to be concerned anymore. Furthermore, users can enjoy a higher quality and seamless service if the connectivity may transit without the handoff latency.

- Handoff Latency and Link Stability: As far as we know, most MNs only use one network interface. In dense wireless environment, MNs may continuously receive wireless signals, but there must be distinguishable handoff latency without any technical enhancement. Meanwhile, because of the lack of the condition from the next wireless network that MNs will handoff to, the stability of next connectivity is unpredictable and then the quality of ongoing service 
may deteriorate. Soft handoff is one solution to eliminate handoff latency and possible instability if we use multi-interface techniques such as MCoA, or Simultaneous Mobility Binding (SB-MIP) [17] which means that MN may use simultaneous mobility binding with more than one network devices to avoid packet loss and discontinuity during the moving process. In addition, the $\mathrm{MN}$ can collect the quality information of the next network it might handoff to from the alternative interface, and then determine which access point or base station has better signal quality and whether the MN can obtain a better wireless link than current one after handoff $[18,19]$.

- Bandwidth Exhaustion: Considering handoff latency above, SB-MIP and MCoA seem to be the probable solution. If we use SB-MIP, MNs will concurrently use both interfaces for a long time and exhaust redundant bandwidths under high density network environment. As to $\mathrm{MCoA}$, only one transmission path is in use during overlapped area. MNs can choose the proper BS more flexible and dramatically decrease bandwidth exhaustion.

- CoA Binding Trails:

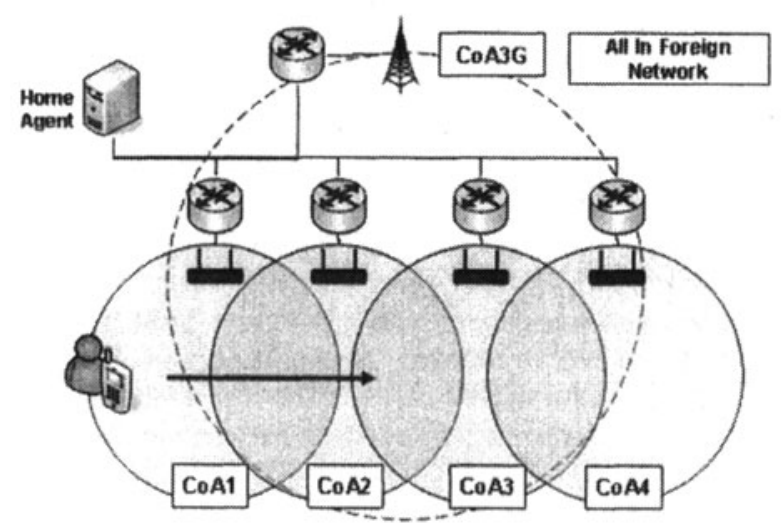

Fig. 8. Linking Map [14]

In Figure Fig. 8, suppose all networks are in different IP domains. MNs probably have an IP variation like CoA1->CoA2->CoA3->CoA4 or CoA 1->CoA3G$>\mathrm{CoA} 4$ during the moving process. It makes no difference in SB-MIP due to simply IP address recording but distinguished by MCoA with layer 2 devices binding unique identification number (BID) which is an identification number to distinguish MN's multiple registrations that CoA3G binds an interface with distinct bandwidth. By this kind of linking characteristics, MN may choose CoAl->CoA3G->CoA4 IP variation for bandwidth conservation with more handoffs, or directly link to CoA3G to lower handoff frequency, and handaround may help us to achieve that. However, the performance comparison of these two binding trails is out of this paper's scope and also an open problem. 
Hand-around will inevitably be one procedure in the future wireless network on the trend of denser BS' distribution. MNs with multi-interface may eliminate handoff latency. MCoA and SB-MIP are two mechanisms of multi-interface management. Moreover, next the following subsection will show MCoA can save more bandwidth than SB-MIP under highly overlapped wireless network environment. We suggest that $\mathrm{MCoA}$, with the ability of interface identification, should be the better one.

\subsection{SIMULATION AND RESULTS}

We simply analyze the handoff latency and bandwidth exhaustion about MCoA. We use NS2-2.28 plus MobiWan module to simulate MCoA procedure and use IEEE $802.11 \mathrm{~b}$ configuration for wireless network connection.

In the part of MCoA module, we modify MobiWan and refer to [20] about the BU message configuration. Since the draft doesn't define any interface switching timing guideline, we initiate interface switching procedure in the meanwhile moving into the wireless overlapped area.

Table 1. Simulation parameters of handoff latency

\begin{tabular}{|c|c|}
\hline Network Simul ator & NS2-228+MobiWan \\
\hline Simulation Environment Scope & $600 \times 50(\mathrm{~m} 2)$ \\
\hline BS Radius & $109(\mathrm{~m})$ \\
\hline BS1 Position & $0(\mathrm{~m})$ \\
\hline BS2 Position & $150(\mathrm{~m})$ \\
\hline Scope Between BSs & $150(\mathrm{~m})$ \\
\hline Simulation Time & $75(\mathrm{~s})$ \\
\hline MN Start Position & $0(\mathrm{~m})$ \\
\hline MN Speed & $2(\mathrm{~m} / \mathrm{s})$ \\
\hline Wireless MAC & $802.11 \mathrm{~b}$ \\
\hline Interface Queue & DropTail \\
\hline Transport Layer & TCP \\
\hline Application Layer & FTP \\
\hline Packet Data Size & $1000(\mathrm{bytes})$ \\
\hline RA Interval & $30 \sim 70$ (ms) \\
\hline
\end{tabular}

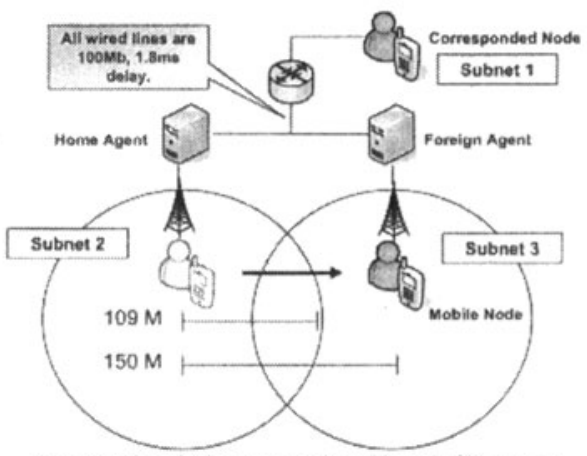

Fig. 9. Simulation scenario of handoff latency 


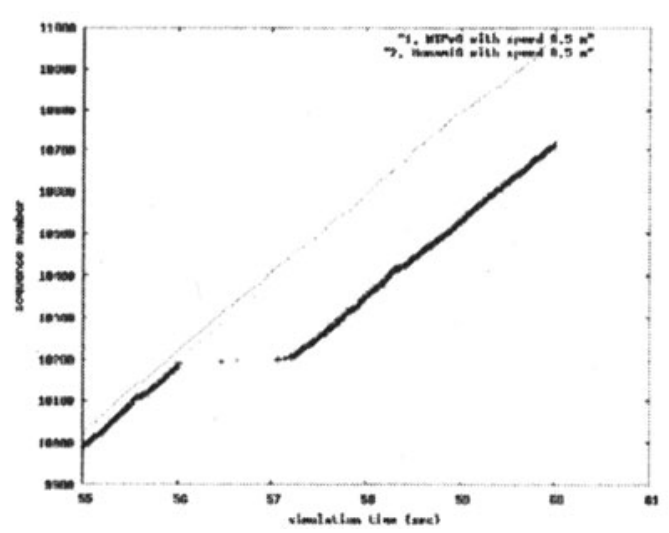

Fig. 10. Handoff Latency between MIPv6 and MCoA [14]

Table 1 and Fig. 9 are the arguments and environment for the Simulation scenario of handoff latency. In Fig. 10, MN processes a handoff procedure between two wireless overlapped BSs. We can figure out that $\mathrm{MCoA}$ has nearly no packet transmission delay resulted from the make-before-break handoff procedure. The MN with MIPv6 module has about 1.26s latency because MIPv6 detects and initiates handoff only when receiving routing advertisement from new V6 router or BU registration timeout. This handoff latency should add extra one second according to Duplicate Address Detection (DAD) [21] since MobiWan doesn't implement DAD operation. However, it makes no difference in MCoA benefited from soft handoff procedure. This means multi-interface mechanism really has better performance on handoff latency.

Table 2. Simulation parameters of bandwidth consumption

\begin{tabular}{|c|c|}
\hline Network Simul ator & NS2-228 \\
\hline Simulation Environment Scope & $600 \times 50(\mathrm{~m} 2)$ \\
\hline BS Radius & $109(\mathrm{~m})$ \\
\hline BS1 Position & $0(\mathrm{~m})$ \\
\hline BS2 Position & $200(\mathrm{~m})$ \\
\hline Scope Between BSs & $200(\mathrm{~m})$ \\
\hline Simul ation Time & $40(\mathrm{~s})$ \\
\hline MN Start Position & $80(\mathrm{~m})$ \\
\hline MN Speed & $1(\mathrm{~m} / \mathrm{s})$ \\
\hline Wireless MAC & $802.11 \mathrm{~b}$ \\
\hline Interface Queue & DropTail \\
\hline Transport Layer & UDP \\
\hline Application Layer & CBR \\
\hline Packet Data Size & $1500(\mathrm{bytes})$ \\
\hline RA Interval & $1(\mathrm{~s})$ \\
\hline
\end{tabular}




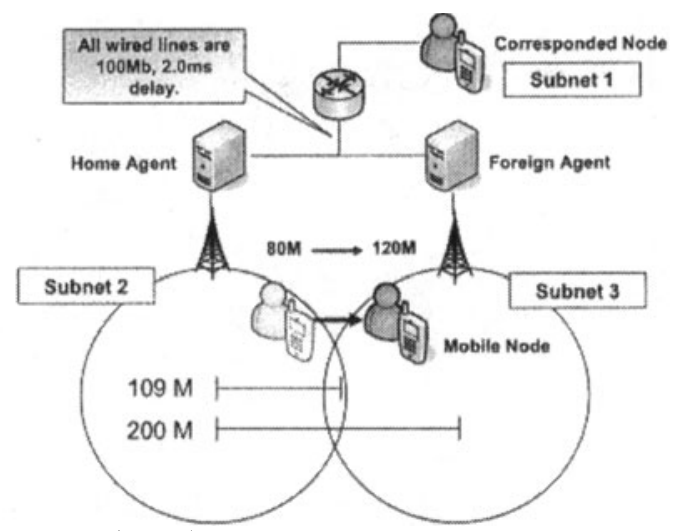

Fig. 11. Simulation scenario of bandwidth consumption

Table 2 and Fig. 11 are the parameters and environment for the Simulation scenario of bandwidth consumption. In Fig. 12, we compare bandwidth exhaustion between two BSs by measuring throughputs on MN. We modify the MIP module in NS2 for SB-MIP and duplicate the packets when moving into the overlapped area. The MN with SB-MIP module has twice bandwidth exhaustion of the original $1 \mathrm{M} / \mathrm{s}$ constant bit rate (CBR) traffic at about 18 secs in the figure. The other $\mathrm{MN}$ with $\mathrm{MCoA}$ module has an unstable transmission bit rate during the handoff procedure but maintains a $1 \mathrm{M} / \mathrm{s}$ bit rate most of the time. We conclude that SBMIP exhausts more bandwidth in the overlapped area than MCoA.

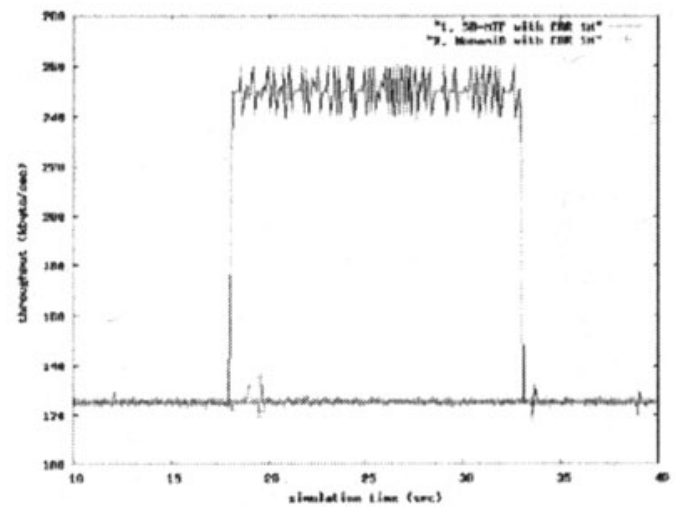

Fig. 12. Multi-interface bandwidth exhaustion between SB-MIP and MCoA[14]

Because MCoA has lower handoff latency than MIPv6 by using soft handoff method in our simulation and takes less bandwidth than SB-MIP in the overlapped area, we conclude that $\mathrm{MCoA}$ should be the most proper layer 3 mechanism for hand-around. 


\section{Hand-around in Home Networking}

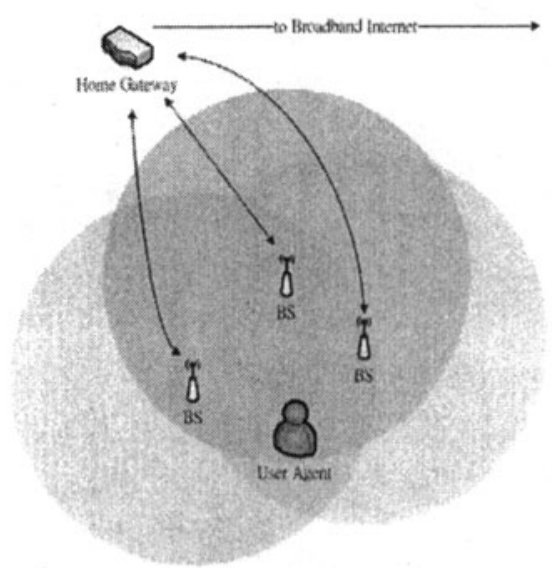

Fig. 13. Wireless Home Networking

Now we introduce multiple care-of address (MCoA) and hand-around notions to home networking. The home networking shown in Fig. 13 supports mobile IP with the two notions. By using MCoA, the mobile node can connect to foreign networks via base stations; and hand-around mobile IP techniques could handle the traffic tunneling. This design can achieve seamless handoff even if the application doesn't support. Moreover, home gateway is an important existence in home networking, and its standing is similar to home agent since they both play an administrative role. So we suggest that the home agent should be placed on the home gateway.

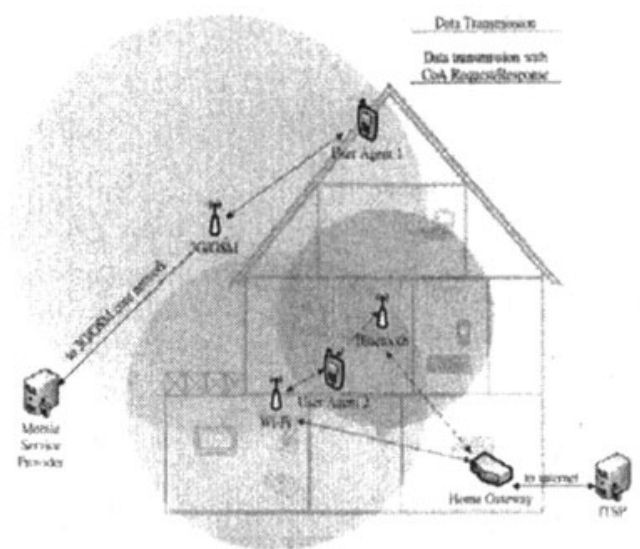

Fig. 14. UMA/Multi-mode Cell Phone Applications with MCoA 
Fig. 14 shows two cases of using MCoA on UMA or multi-mode cell phones with Wi-Fi and Bluetooth capabilities. User agent 1 is not at home, or can't receive good wireless signals of home networking. So user agent 1 uses 3G/GSM network to establish or maintain call sessions. User agent 2 , who has came back home in the house, receiving bad or even no signal from 3G/GSM base stations, uses internet and VoIP via Wi-Fi or Bluetooth with better signal quality to maintain the call session. In this case, User agent 2 can also switch between Wi-Fi and Bluetooth. User agent 2 is currently using Wi-Fi as shown in Fig. 14, but it also maintains a CoA with the Bluetooth network. And if the signal quality of Bluetooth becomes much better than Wi-Fi while moving, user agent 2 just simply switches to the CoA in the Bluetooth network instead of Wi-Fi. This kind of handoff, or what we called hand-around can achieve seamless. To the user who is talking on phone, the call session won't be disconnected or interrupted so the user don't even aware of handoffs; to the internet telephone service provider (ITSP) [22], the service protocols don't need to be substantially modified for mobility and seamless concerns, since these protocols are all based on IP, which is a layer-3 protocol and independent of the ITSP's protocols.

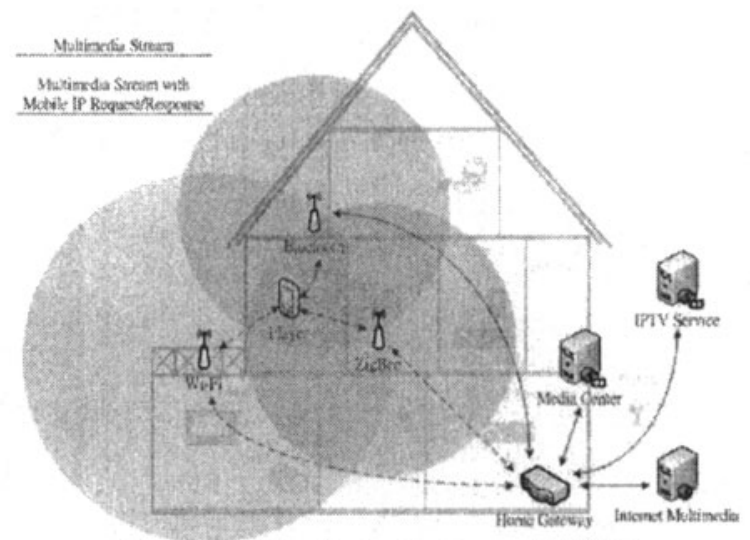

Fig. 15. Multimedia Applications with $\mathrm{MCoA}$

Fig. 15 shows that multimedia services and applications can also be improved by MCoA and hand-around notions. Whether the multimedia clips and streams came from the internet or the media center at home, as long as the player carried by, or placed near the user, these media streams must go through the home gateway. On the other hand, since the home gateway could also be the home agent of mobile IP network, the media streams can be tunneled to the CoAs. We assume the media player with multiple connectivity uses Bluetooth to receive media streams in the beginning. When the signal of Bluetooth is turning too weak to maintain the media quality, the player should try another CoA that offers connectivity which could reach the quality and seamless requirements of multimedia. Thus, the user can enjoy the multimedia entertainment without breaking off and quality loss, even if the user is on the move in the house as long as wireless connectivity exists. 
The multimedia service providers, such as the media center at home, IPTV, and other internet media sources, need no revision and redirection.

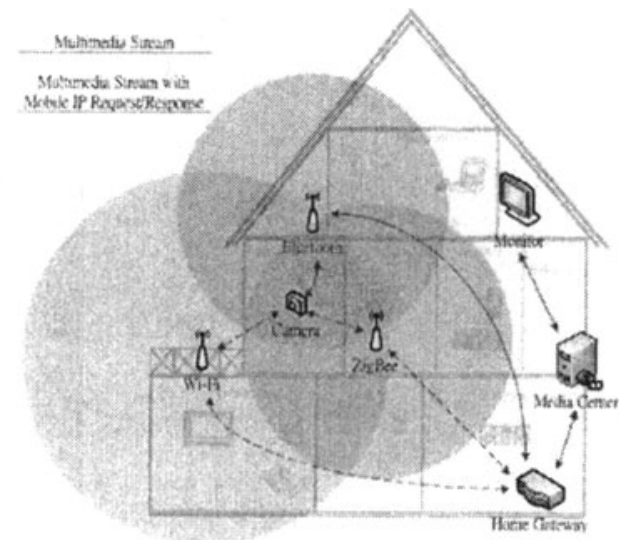

Fig. 16. Security Applications with $\mathrm{MCo} A$

Fig. 16 shows a similar case comparing to Fig. 15, substituting the multimedia player to a camera. Just like we stated to the multimedia player in the last paragraph, the camera is enabled with mobility, so the changing of position won't affect the continuity of transmitting and recording. Furthermore, we can collocate with the multimedia playing applications, and create a security service by dynamic monitoring. For example, a baby can crawl all over the house to satisfy her curiosity, and may put herself in danger. Adults can attach the camera to the baby, and carry a multimedia player as the monitor. Wherever the baby goes, with available wireless connectivity, the adults can keep a watch on her every second even if the adults themselves are moving in the house. This is just an example. While putting $\mathrm{MCoA}$ and hand-around mobility notions into home networking, there must be further services and applications to invent, improving our lives from various aspects and dimensions. 


\section{Conclusions}

Hand-around is a new paradigm of connectivity transition with heterogeneous networks and no handoff latency. It can manage multiple interfaces to accomplish seamless connections and mobility. Home networking has some characteristics matching the observations of hand-around, such as a small space crowded with wireless base stations, highly overlapped wireless coverage, and concurrent activation of multiple interfaces. So, to home networking, hand-around not only brings performance advancing but also provides support of seamless services and mobility. With hand-around, the picture of digital life will be more complete and convenient.

\section{Acknowledgement}

This work was supported partially by National Science Council of Republic of China under Grants NSC: 96-2219-E-194-008.

\section{References}

1. Bluetooth; http://www:bluetooth.com/.

2. Wi-Fi; http://www.wi-ti.org/ .

3. ZigBee; http://www'zigbec.org/.

4. IrDA; http://www.irda.org/ .

5. wireless USB; http://www.usb.org/developers/wusb/.

6. WiMedia; http://www.wimedia.org/ .

7. Home Gateway Technical Requirements: Release 2; http://www.homegatewayinitiative.com.

8. VoIP; http://en.wikipedia.org/wiki/Voip .

9. UMA; http://www.umatoday.com/ .

10. pervasive gaming; http://en.wikipedia.org/wiki/Pervasive_game .

11. IPTV; littp://www.itu. int/ITU-T/IPTV/.

12. Elin Wedlund and Henning Schulzrinne, Mobility support using SIP, Pages: $76-82$ (1999).

13. R. Wakikawa, T. Ernst, K. Nagami, Multiple Care-of Addresses Registra-tion, draft-ietfmonami6-multiplecoa-02.txt, March 5, 2007

14. Jing-Luen Lin and J.-Y. Pan,Hand-around: A Handoff Evolution with Monami6, (WiCOM Shanghai 2007).

15. M. Johnsson, J. Sachs, T. Rinta-aho, and T. Jokikyyny, Ambient Networks -A Framework for Multi-Access Control in Heterogeneous Networks, IEEE 64th Vehicular Technology Conference, pp. 1-5 (2006).

16. N. Akhtar, M. A. Callejo, and J. A. Colas, Ambient networks, an architecture for communication networks beyond $3 G$, IEEE 61 st Vehicular Technology Conference, pp. 2974-2978 (2005).

17. C. Perkins, IP Mobility Support for IPv4, RFC 3344, Aug 2002.

18. T. Min, T. Lin, and K. Jianchu, A seamless handoff approach of mobile IP based on dual-link, Proceedings of the First International Conference on Wireless Internet, pp. 5663 (2005). 
19. R. Inayat, R. Aibara, and K. Nishimura, A seamless handoff for dual-interfaced mobile devices in hybrid wireless access networks, 18th International Conference on Advanced Information Networking and Applications, pp. 373-378 (2004).

20. M. Kuparinen, H. Mahkonen, and T. Kauppinen, Multiple CoA Per-formance Analysis, draft-kuparinen-monami6-mcoa-performance-00.txt, 2006.

21. T. Narten, E. Nordmark, and W. Simpson, Neighbor Discovery for IP Version 6 (IPv6), RFC 2461, 1998.

22. ITSP; http://en.wikipedia.org/wiki/lTSP. 